\title{
Using shock waves to improve the sound absorbing efficiency of closed-cell foams
}

\author{
Olivier Doutres ${ }^{1}$, Noureddine Atalla ${ }^{1}$, Martin Brouillette ${ }^{2}$, Christian Hébert ${ }^{2}$ \\ ${ }^{1}$ GAUS, Department of mechanical engineering, Université de Sherbrooke (Qc), Canada, J1K 2R1 \\ ${ }^{2}$ Shock Wave Laboratory, Department of mechanical engineering, Université de Sherbrooke (Qc), \\ Canada, J1K 2R1
}

Corresponding author: olivier.doutres@usherbrooke.ca 


\begin{abstract}
Producing closed-cell foams is generally cheaper and simpler than open-cell foams. However, the acoustic and filtration efficiency of closed-cell foam materials is generally poor because it is very difficult for fluid or acoustic waves to penetrate into the material. A new method using shock waves to remove the membranes closing the cell pores (known as reticulation) and thus to improve the acoustic and filtration behavior of closed-cell foam material is presented. Various shock treatments have been carried out on polyurethane and polyimide foams and the following conclusions were drawn: (1) reticulation efficiency increased and thus the airflow resistivity and tortuosity decreased when increasing the amplitude of the shock treatment; (2) the rigidity of the foam is decreased; (3) the process is reliable and repeatable and (4) obtained acoustic performance is comparable to classical thermal reticulation.
\end{abstract}

\title{
Keywords
}

Porous material; Open-cell foam; Reticulation; Shock wave; Sound absorption; Filtration; Airflow resistivity 


\section{Introduction}

Foam microstructure can be seen as a collection of interlinked struts forming a packing of cells interconnected to others through pores. Materials with the totality of pores closed by thin membranes are called closed-cell foams. The filtration and acoustic efficiency of closed-cell foams is poor compared to open-cell foams since it is very difficult for the fluid or the acoustic waves to penetrate inside the material. To improve the filtration and acoustic behavior of closed-cell foam, the membranes closing the cell pores are removed in a process known as "foam reticulation.” Materials having only open pores are called "fully reticulated," while if some of the pores are closed or partially closed, the material is "partially reticulated." Two main reticulation methods commonly used in the foam industry are thermal [1,2] and chemical [3] reticulation. The thermal method involves placing a bun of foam in a very large vessel filled with a combustible gas mixture. The gas is ignited and a controlled flame front passes through the foam, melting the window membranes. However, this method only applies to cellular material having heat destructible membranes. Moreover, it may not be applied in a continuous process and the materials must be cooled after treatment. The chemical reticulation method involves subjecting the foam to a caustic bath which dissolves the window membranes. Chemical concentration, bath temperature and time of exposition of the material in the bath may be controlled accordingly. However, this method results in an expensive process, may use hazardous materials and can produce a strong inhomogeneity of the surface and the volume of the treated material.

A reticulation method dedicated to foams comprising a flexible frame is investigated in this paper. It is based on the impact of a shock wave (i.e., a high-amplitude short-duration 
pressure wave) on the foam surface. This impact involves (i) the propagation of elastic and plastic waves within the porous frame and (ii) a large frame deformation [4,5]. This shockfoam interaction is used in this work to rupture the membranes closing the foam cells [6]. The shock wave reticulation method offers a number of important capabilities, as compared to the aforementioned commercially available systems: (1) the reticulation rate of the treated foam can be tuned depending on the shock strength, (2) the treatment can be varied upon the foam surface to create "acoustic patches" or transverse flow resistance variation effects, (3) it can be applied rapidly and easily in an assembly line, (4) it does not involve chemical products and/or immersion of the foam in a fluid or hot gas, and thus does not require drying or cooling the foam after treatment and (5) it is inexpensive to perform.

This paper presents an experimental validation of the proposed shock wave reticulation method. Seven different foams are reticulated using shock waves: one polyimide and six polyurethane foams. First, the experimental setup and the shock wave generator are described. The seven foams are then presented. The foams microstructures are analyzed before and after treatment from micrographs using light-microscopy or electron microscopy techniques. The reticulation efficiency due to the shock impact and the influence of the shock strength are evaluated from airflow resistivity, tortuosity, stiffness and sound absorption measurements. Finally, the reticulation efficiency of the proposed shock method is compared to the one of the thermal reticulation method on four polyurethane foams. 


\section{Experimental setup}

A shock tube is used to generate controlled shock waves in a gaseous medium. Both the shock tube and the foam are in the same gaseous environment, in the present case, air at room conditions. A simple shock tube uses two tube sections and is illustrated in Fig. 1. The socalled driver section of the shock tube is filled with a high pressure gas from an external supply. A great amount of energy is thus accumulated in the driver tube. The driver tube is separated from a driven or test tube via a membrane; this driven tube is filled with low pressure gas (here, air at atmospheric conditions). When the partition is suddenly removed or ruptured, a shock wave is generated and propagates in the secondary tube toward the closedcell material face placed at the end. A precise control of the driver pressure at rupture is required to generate shock waves of desired strength. It was found that the major controlling parameter of the shock treatment is the Mach number $\left(M_{s}\right)$ of the shock wave. The amplitude of the shock (i.e., the treatment strength) increases with the Mach number. Several parameters can be modified to control the Mach number of the shock wave. They are: gas pressures, gas species, lengths of the high pressure and low pressure sections of the tube, filling time of the high pressure gas and temperature. In this work, only the gas pressure in the high pressure section is varied to change the Mach number and thus the treatment's strength. The gas used in the high pressure section is nitrogen. The pressure is varied from 50 psi to 300 psi to produce Mach numbers ranging between 1.31 and 1.86. The shock tube has circular crosssection with an inner diameter of 1.5 inches. Because the effect of the impact of a shock wave with a flexible material is not homogeneous along the material thickness [4,5], all samples presented in this paper are treated on both sides with similar shock amplitude in order to get samples as homogeneous as possible. 


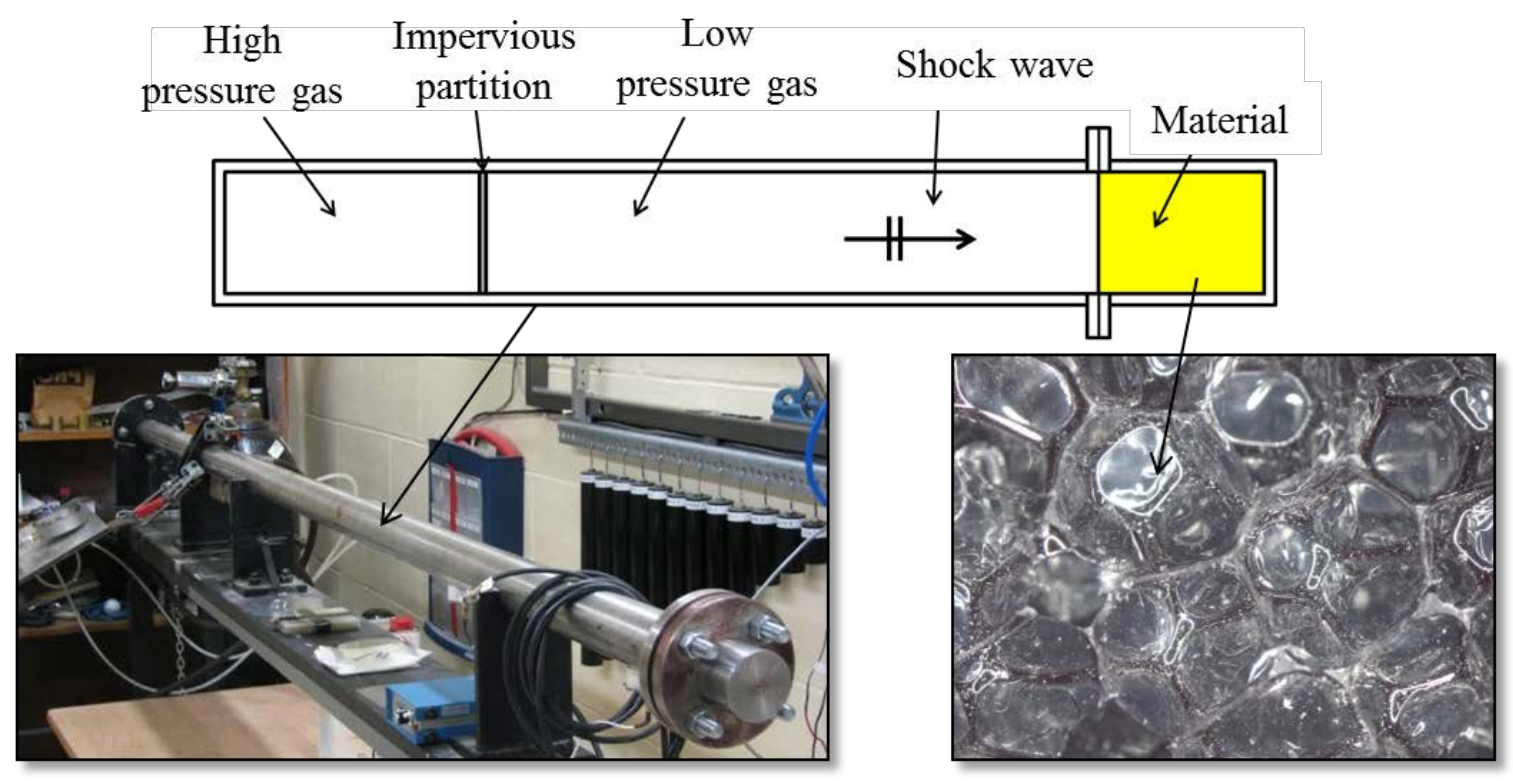

Figure 1

\section{Materials and measurement methods}

\subsection{Materials}

Six different polyurethane foams (N1-N6) and one polyimide foam (N7) are treated using the proposed reticulation method. Polyurethane materials consist of numerous individual cells which generally are constructed of a three dimensional skeletal structure of interconnected struts with membranes joined to the skeletal structure. The skeletal structure in these cellular materials is usually considerably thicker than the membranes or windows [1]. This 3D microstructure can be idealized as a packing of tetrakaidecahedra cells interconnected through pores $[7,8]$. The cell size in ppi (ppi=pore per inch) of foams N1 to N6 varies between 8 ppi and 80 ppi. The cell struts have a triangular concave cross-sectional shape which is nonuniform along the strut length; the cross section is smaller at the center of the strut. Thus, the intersections between struts, also called nodes, are zones of material concentration [9]. The 
cell pores can be open or closed by thin membranes as mentioned previously. The reticulation rate $R_{w}$ defined in references [7,8] quantifies the open pore content within the porous aggregate and can be used as an estimate of the cell interconnectivity. Fully reticulated foams have a reticulation rate of $100 \%$. This microstructure parameter has not been estimated in this work but it is expected to be less than $10 \%$ for all base materials (i.e., untreated materials). For example, figures 2(a), 2(d) and 2(g) present micrographs of the three untreated polyurethane foams N1, N2 and N4 taken with the help of a stereo-microscope Leica MZ6 or a scanning electron microscope (SEM) Hitachi S-3000N. These figures clearly show that most of the pores are closed by the thin membranes.

Material N7 is a polyimide foam, known for its unique combination of superior fire resistance, low smoke and virtually no toxic gas emission, wide operating temperature range and low density $[10,11]$. The microstructure of the untreated polyimide foam N7 is also captured using an SEM microscope and is presented in Figs. 3(a) and 3(b). The microstructure of the foam is constituted of large cells connected to each other by thin membranes $(\approx 15 \mu \mathrm{m}$ thick). Even if polyimide foam appears to be closed-cell type (as shown in Figs. 3(a) and 3(b)), the thin membranes are not connected all together which makes polyimide foams highly porous foams but with low cell-interconnectivity. Consequently, porosity and airflow resistivity of the untreated polyimide foams are $\phi=95 \%$ and $\sigma=800000$ N.s.m ${ }^{-4}$ respectively. 


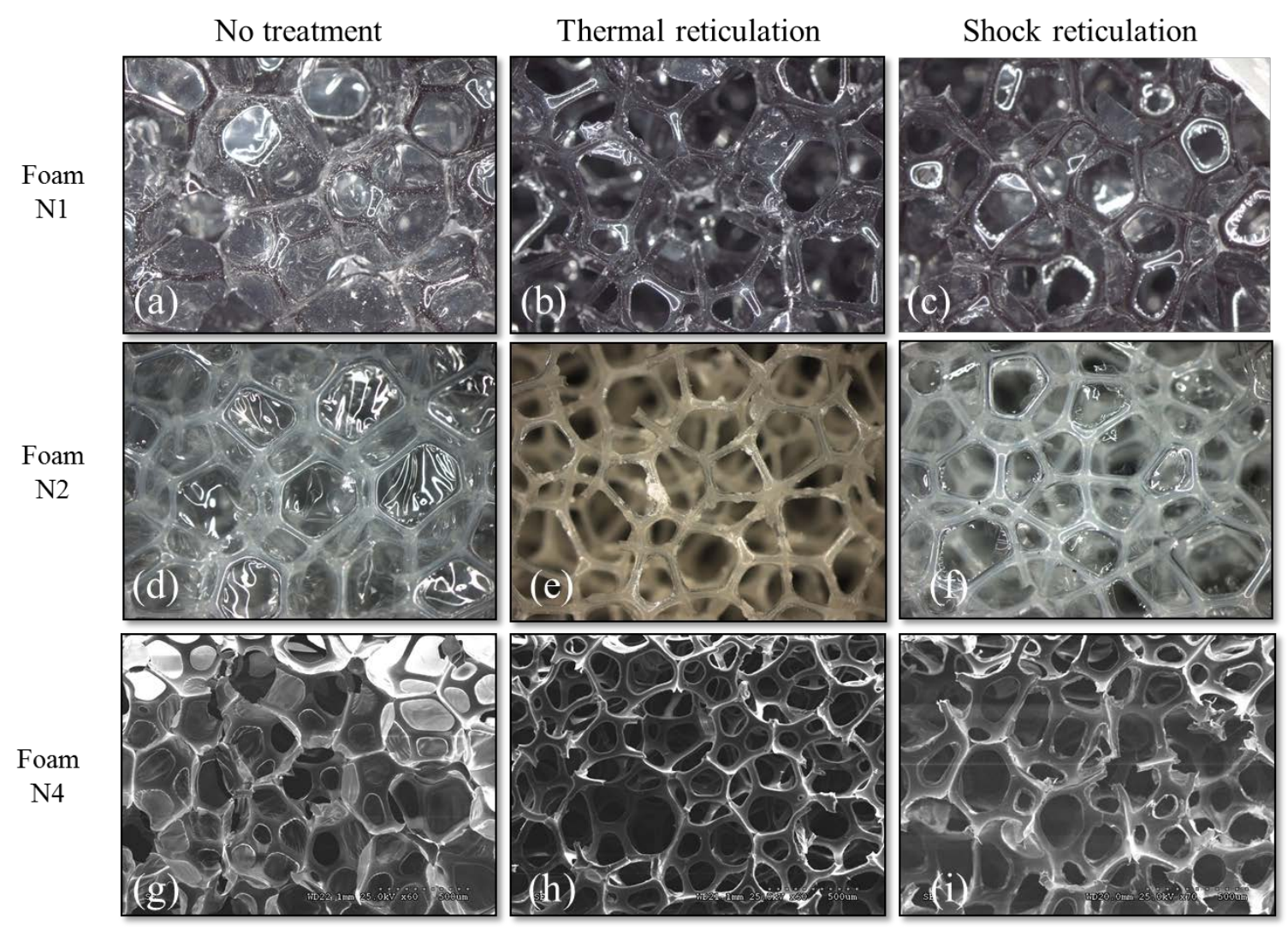

Figure 2 

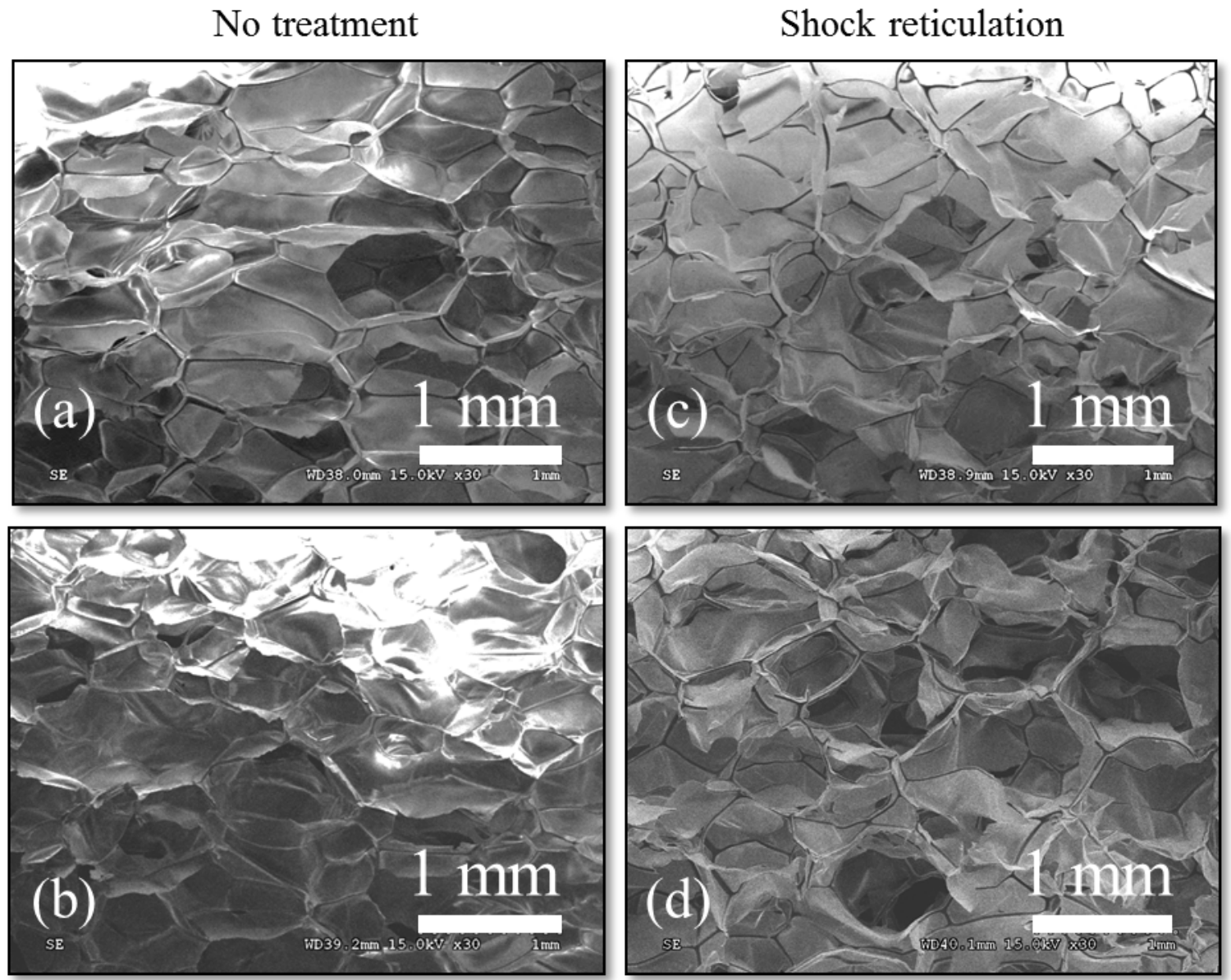

Figure 3

All samples are precisely die-cut using a rotary drill press. The internal diameter of the cutter is chosen to provide samples with external diameter $0.5 \mathrm{~mm}$ less than the impedance tube diameter (see sec. 3.2.4) as suggested in [12] and [13]. All treated samples are approximately 1 inch thick. 


\subsection{Measurement methods}

\subsubsection{Volume and density}

The dimensions of the cylindrical samples are measured according to the standard ASTM D-3574 [14] (see sec. 8) using a sliding caliper gage. The density of the samples is simply determined according to standard ASTM D-3574 test A from the ratio of the sample mass to the sample volume. The mass of the samples is measured using an Ohaus Explorer balance, model no. E14130.

\subsubsection{Mechanical properties}

The effect of the shock treatment on the mechanical properties of the polyimide material is investigated from compression tests carried out at low frequencies using a quasi-static method (QMA) [15,16]. This test method is based on the measurement of the mechanical impedance of a cylindrical sample placed between two rigid plates and subjected to a small amplitude sinusoidal compression. Two samples of the base material having different shape factors (i.e., half the radius to thickness ratio of the sample) are used to get both Young's modulus and Poisson's ratio according to the method proposed by Langlois et al. [16]. Samples reticulated by the shock wave treatment have only one shape factor (the thickness is 1 in. and the sample diameter is $1.5 \mathrm{in}$. which is set by the shock tube where the samples are treated). The Young's modulus is then simply determined from compression stiffness measurements, considering that the Poisson's ratio remains unchanged (that is equal to the one of the untreated material). During all compression tests, a static strain of $1 \%$ is applied to the sample and a dynamic strain less than $0.05 \%$ to avoid non-linearity effect [17]. 


\subsubsection{Airflow resistivity and tortuosity}

Airflow resistivity and tortuosity are two intrinsic parameters commonly used in models that describe wave propagation in porous media such as the Johnson-Champoux-Allard (JCA) model [18-20]. These two parameters are used in this work as a measure of the reticulation treatment efficiency since it has been shown elsewhere $[7,8]$ that their value decreases with an increasing amount of broken membranes (i.e., open pores).

The airflow resistivity $\sigma$ of acoustical materials is the airflow resistance $R$ divided by the sample thickness. The airflow resistance is defined as the quotient of the air pressure difference across a sample divided by the volume velocity of airflow through this sample and it is measured in the present work according to the ISO 9053 standard [21]. This parameter, which links the macroscopic fluid velocity to the imposed pressure gradient according to Darcy's law, is particularly relevant to describe the viscous interaction between the frame and the saturating fluid at low frequencies (i.e., when the viscous skin depth is larger or in the order of magnitude of the pore size). It is worth noting that the static permeability $k_{0}\left(k_{0}=\eta / \sigma\right.$, with $\eta$ the viscosity of the saturating fluid) is often used instead of the airflow resistivity, in particular by geophysicists, since it does not depend on the saturating fluid but only on the material frame micro-geometry.

The tortuosity $\alpha_{\infty}$ is another intrinsic parameter related to the complexity of the frame micro-geometry. It is used in the models $[18,20]$ to account for the inertial interaction between the frame and the saturating fluid at high frequencies (i.e., when the viscous skin depth is small compared to the pore size and the viscosity effects become negligible), and thus depicts an added mass effect of the saturating fluid such as $\rho=\alpha_{\infty} \rho_{0}$ with $\rho$ the effective density 
of the fluid and $\rho_{0}$ the density of the saturating fluid. It is often interpreted as a characteristic of the sinuous aspect of the fluid flow associated with the passage of a wave in a porous media. The tortuosity always has a value greater than 1 . In the present study, the tortuosity of the foams is estimated from the measurement of acoustic waves reflected by a slab of porous material at oblique incidence using the ultrasound technique proposed by Fellah et al. [22].

\subsubsection{Sound absorption coefficient}

The sound absorption coefficient $\alpha$ is related to the reflection coefficient $r$ defined as the ratio of the pressures created by the outgoing and ingoing waves at the surface of the layer: $\alpha=1-|r|^{2}$. The normal incidence sound absorption coefficient is measured according to standard ASTM E1050-10 [13] using a 1.5 in diameter impedance tube. It is important to note that the sound absorption coefficient is not a characteristic of the treatment efficiency. Indeed, the sound absorbing efficiency can be decreased by the reticulation process if the airflow resistivity of the untreated sample is already close to its optimum value [20,23].

\section{Results and discussion}

\subsection{Effect on the microstructure}

Figure 2 presents several photo-micrographs of PU foams N1 and N2 and SEMmicrographs of PU foam N4 taken before and after reticulation. In this case, the shock reticulation method is applied on base materials using a Mach number of 1.8. Note that the selection of this Mach number is based on the optimum treatment for foam N5 (see section 4.2). Foams N1, N2 and N4 have an important amount of closed pores as shown in Figs 2(a), 
2(d) and 2(g). Figs. 2(c) and 2(f) show that, after the shock treatment, the membranes of PU foams N1 and N2 with large cell size (10 and 8 ppi respectively) are punctured and stay attached to the pores. In the case of the PU foam N4 with small cells (i.e., 80 ppi), Fig. 2(i) clearly indicates the great amount of broken membranes and the presence of residual membranes around pores' perimeter. Thermal reticulation has been also performed on the same foams. Figs. 2(b), 2(e) and 2(h) show that the thermal reticulation melts almost all the membranes, with the burnt membranes then sticking around the struts resulting in an increased stiffness of the foam. SEM micrographs of the polyimide material N7 taken before and after a shock treatment of amplitude $M_{s}=1.62$ are presented in Fig. 3. Contrary to PU foams, the microstructure of the polyimide foam treated with this shock amplitude ( $\left.M_{s}=1.62\right)$ seems barely affected by the shock treatment (see Figs. 3(c) and 3(d)). Few broken membranes or open pores can however be seen in Fig. 3(d) but most of the membranes are still present. Mechanical strength of membranes appears to be more important in the case of polyimide foams compared to polyurethane foams. For higher shock treatment (i.e., $M_{s}>1.7$ ), the polyimide foam is subjected to a large permanent compression; struts and remaining membranes are brought nearer from each other. This strong modification of the microstructure will result in large modification of the macroscopic properties as detailed in the next section.

\subsection{Influence of shock strength}

The influence of the shock strength is investigated on PU foam N5 and polyimide foam N7. The two foams are subjected to a shock treatment with increasing strength: from $M_{s}=1.35$ 
to $M_{s}=1.85$ for foam N5 and from $M_{s}=1.31$ to $M_{s}=1.86$ for foam N7. As mentioned previously, each sample is treated on both sides with identical shock wave strengths.

The thickness of the material $h$ decreases with increase the shock strength, as shown in Figs. 4(a) and 5(a). This decrease is only significant for large shock strengths $\left(M_{s}>1.5\right.$ for both foams). Indeed, for strong shocks, the porous frame is compressed when the shock wave impacts onto the porous surface [4,5], and foam compression can be sufficiently important to reach plastic deformation. The permanent compression is defined as $h_{c}=100 \times\left(h_{0}-h\right) / h_{0}, h_{0}$ being the thickness of the sample before treatment. For the polyurethane foam N5, this effect is not significant and permanent compression reaches a maximum of $10 \%$ for $M_{s}=1.85$ (see Figs. 4(a)). Permanent compression of polyimide foam N7 is low for $M_{s}<1.58\left(h_{c}<10 \%\right)$ and increases considerably for higher shock strengths, reaching a maximum of $h_{c}=55 \%$ for $M_{s}=1.86$. This decrease in thickness results in an increase of the foam density since the mass of the sample is not modified by the shock treatment (see Figs. 4(b) and 5(b)).

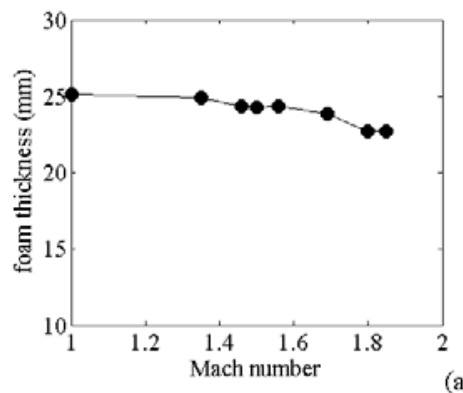

(a)

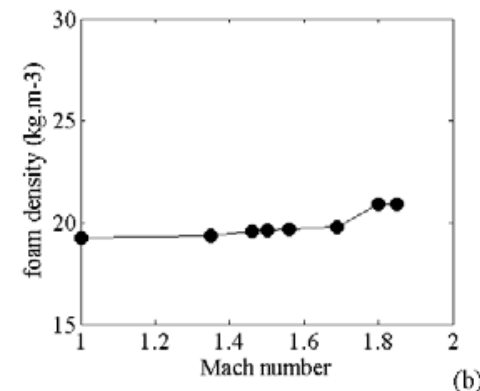

(b)

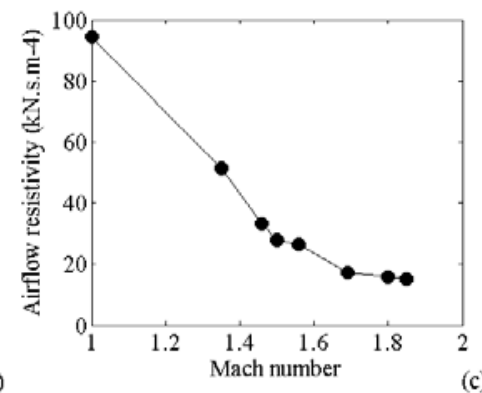

(c)

Figure 4

As expected, airflow resistivity decreases with increasing shock strength (see Figs. 4(c) and 5(c)) while the amount of broken membranes increases. For the case of foam N5, at first 
the decrease is quite rapid, and afterwards a plateau seems to be reached for $M_{s}>1.7$. The resistivity of the base material is reduced by a factor of 6.3 for $M_{s}=1.85$. The airflow resistivity decrease for the polyimide foam N7 is even greater; it is reduced by a factor of 77 for $M_{s}=1.68$. A plateau can be observed for $1.53<M_{s}<1.73$ (see zoom in Fig. 5(c)). This plateau is followed by an increase of the airflow resistivity, which is due to the large plastic deformation, i.e. large permanent compression, of the polyimide foam for high shock amplitude as shown in Fig. 5(a). Indeed, it is well known that frame compression results in an increase of airflow resistivity and tortuosity since the various struts and remaining membranes are brought nearer from each other $[24,25]$. These results show that the proposed reticulation method can be used to tune the resistive behavior of closed-cell foams depending on the final application of the foam material.
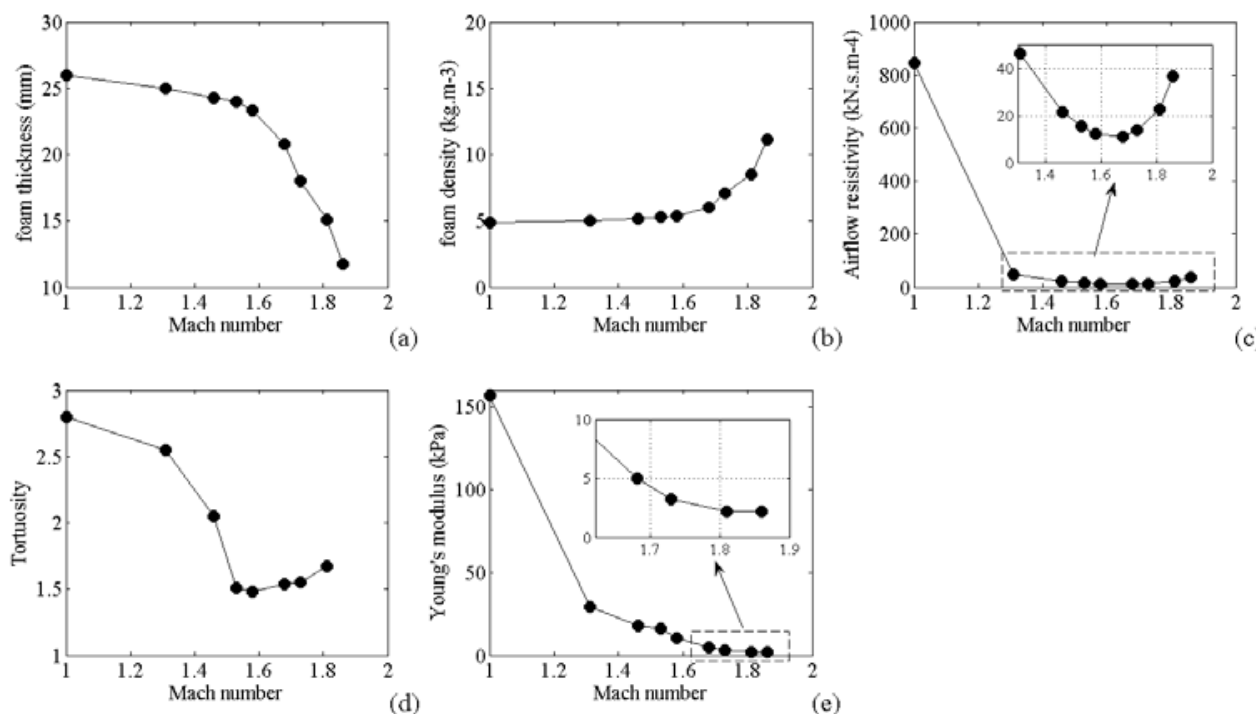

Figure 5 
The variation of the tortuosity with shock strength is investigated for the polyimide foam N7. A similar trend as with the airflow resistivity parameter is observed (see Figs. 5(c) and 5(d)): i.e., a decrease for $M_{s}<1.53$, a plateau for $1.53<M_{s}<1.73$ and an increase for $M_{s}>1.73$. The reduction in tortuosity indicates that the actual path followed by the acoustical wave through the material is more direct (less sinuous). This is clearly associated to the rupture of membranes even if this is barely visible on the SEM micrographs of Figs. 3(c) and 3(d). According to the observations made on PU foams in ref. [7], the tortuosity logically does not reach the minimum of 1 since membranes are still present after reticulation. The tortuosity increase for $M_{s}>1.73$ is, as before, due to the high frame compression. Fig. 5(e) shows that the stiffness of the polyimide frame always decreases as the shock strength increases. The shock wave reticulation method can thus be used to soften porous materials, which could be of particular interest for several applications such as vibration damping, packaging for delicate goods, comfort products (e.g., mattresses, seats, cushions, etc.).

The sound absorption coefficients of the base materials (referred to by "blank" in the figures) and some reticulated materials are presented in Fig. 6 for both types of foams. Their sound absorption averages (SAA) are provided in Table 1. The SAA is defined in [26] as the average of the sound absorption coefficients for the twelve one-third octave bands from 200 through $2500 \mathrm{~Hz}$ (note that this overall rating is similar to the well-known noise reduction coefficient NRC [26]). 

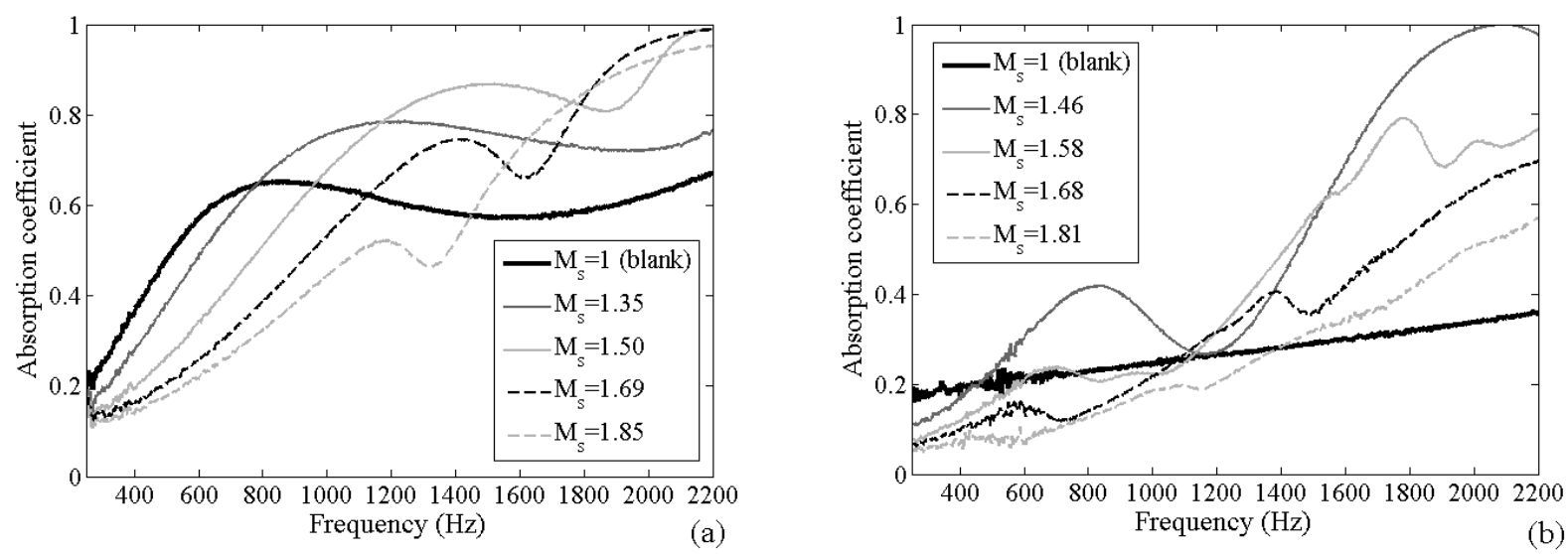

Figure 6

Overall, the low frequency sound absorption decreases and the high frequency sound absorption increases with increasing shock strength. This is expected since an increase in shock strength is associated with an airflow resistivity decrease (see Figs. 4(c) and 5(c)). The frequency transition between the low and the high frequency range is around $1 \mathrm{kHz}$ in this case. The SAA of material N5 is not clearly increased by the shock reticulation treatment because the high frequency improvement compensates for the loss at low frequency. In the case of the polyimide material N7, the shock treatment clearly improves the sound absorption property of the base material, especially for $M_{s} \leq 1.68$ (see Fig. 6(b) and Table 1). It is worth noting that, for $M_{s}=1.81$, the sound absorption is not greatly improved compared to the blank material because the sample thickness is greatly decreased $\left(h_{c}=42 \%\right.$ for $\left.M_{s}=1.81\right)$. Because of this, it is obvious that a foam sample reticulated with $M_{s}=1.81$ and having a final thickness of 1 inch would have a much higher sound absorption coefficient. 


\subsection{Comparison with the thermal reticulation method}

As mentioned in section 4.1, the thermal method melts the membranes whereas the shock method punctured them. For comparison purposes, the four polyurethane foams N1-N4 are reticulated using either the shock technique or the thermal method. The shock treatment is carried out with the impact of a shock wave with $M_{s}=1.80$ on both side of the samples. The reticulation efficiency is evaluated from airflow resistivity measurements, with the results presented in Table 2. Both reticulation methods greatly decrease the airflow resistivity of the base materials. The thermal reticulation appears slightly more efficient but both methods produce materials with airflow resistivities in the same order of magnitude. However, it is worth noting that the shock reticulation applied to these foams, with shock wave having Mach number of $M_{s}=1.80$, has not been optimized. Recall also, that contrary to thermal reticulation, shock reticulation can be applied to foam having membranes that cannot be destroyed by heat. Finally, note that the thermal reticulation method cannot be used for the polyimide foam material N7. The mechanical stress occurring during the gas explosion destroyed the material. According to Evonik Foam, the polyimide foam company who tried out the thermal reticulation on polyimide foams, the same settings classically used for polyurethane foam (i.e., composition of the fuel-gas and oxygen mixture, filling time, etc...) was employed so it is highly unlikely that it was a temperature issue.

\section{Conclusion}

In this paper, a reticulation method based on the impact of a shock wave with a flexible closed-cell or partially closed-cell porous material has been investigated. The method is 
applied to 6 different polyurethane and 1 polyimide foams. An analysis of the foam microstructure showed that the shock impact ruptures the membranes closing the cells pores. The shock wave impact leads to an important decrease of the airflow resistivity, the tortuosity and the stiffness of the foam. All these properties decrease with an increase of the shock strength characterized by its Mach number $M_{s}$. The proposed reticulation method can be used to tune the acoustical behavior of closed-cell or partially closed-cell materials. It appears that, for materials having a rather low elastic deformation range, the airflow resistivity and tortuosity reductions are followed by a slight increase while increasing the shock strength (for $\left.M_{s}>1.6\right)$. This increase of the intrinsic properties is due to the large permanent frame compression associated to the high strength shock treatment. As expected, it was observed that both the medium and high frequency sound absorption behavior of the foams was improved by the proposed reticulation method.

The shock reticulation method is compared to the widely used thermal method. The aforementioned method allows to reticulating closed-cell foam materials by melting the cell membranes. Micrographs of foams reticulated with the proposed shock method show that pores' membranes are ruptured but may still be attached to the cell pores. The reticulation efficiency is found similar but slightly lower compared to the thermal method due to the remaining membranes. However, this property can be of particular interest for filtration purposes since the shock method greatly decreases the airflow resistance of the material but keeps a complex skeletal structure which is ideal for trapping dust and particles. More importantly, the proposed reticulation method allows for a control of the reticulation rate upon the foam surface to create "acoustic patches" or transverse flow resistance variation effects 
and it can be applied rapidly and easily in an assembly line. This is not possible in the thermal method.

\section{Acknowledgements}

The Woodbridge Foam Corporation and Evonik Foams are gratefully acknowledged for supplying all materials. The authors are also grateful to M. Charlebois-Ménard for his contribution to this work. Finally, the authors would like to thank the National Sciences and Engineering Research Council of Canada (NSERC) for providing financial support.

\section{References}

1. Geen HC, Arbor A, Rice WA. Process for bonding and/or reticulation. United States Patent 1965; 3175025.

2. Meisel FW, Media J, Blair EA. Method for explosion reticulation using a noninflammable liquid. United States Patent 1967; 3297803.

3. Lox WE, Petrich W. Reticulated polyurethane foam and method of making same. United State Patent 1973; 3753756.

4. Seitz MW, Skews BW. Effect of compressible foam properties on pressure amplification during shock wave impact. Shock Waves 2006; 15: 177-197.

5. Petel OE, Ouellet S, Higgins AJ, Frost DL. The elastic-plastic behaviour of foam under shock loading. Shock Waves 2012; DOI 10.1007/s00193-012-0414-7. 
6. Atalla N, Brouillette M, Doutres O, Hébert C. Method and apparatus for reticulating foams using shock waves in a gaseous environment. U.S. Patent Application No. 61/528,921 (filed Aug. 30, 2011).

7. Doutres O, Atalla N, Dong K. Effect of the microstructure closed pore content on the acoustic behavior of polyurethane foams. J. Appl. Phys. 2011; 110: 064901.

8. Doutres O, Atalla N, Dong K. A semi-phenomenological model to predict the acoustic behavior of fully and partially reticulated polyurethane foams. J. Appl. Phys. 2013; 113: 054901.

9. Jang WY, Kraynik AM, Kyriakides S. On the microstructure of open-cell foams and its effect on elastic properties. Int. J. Solids Struct. 2008; 45: 1845-1875.

10. Gagliani J, Supkis DE. Non-flammable polyimide materials for aircraft and spacecraft applications. Acta Astronautica 1980; 7:653-683.

11. Sazanoz YN. Applied significance of Polyimides. Russian Journal of Applied Chemistry 2001; 74(8): 1253-1259.

12. Kino $\mathrm{N}$, Ueno $\mathrm{T}$. Investigation of sample size effects in impedance tube measurements. Appl. Acoust. 2007; 68: 1485-1493.

13. Anonymous. Standard test method for impedance and absorption of acoustical materials using a tube, two microphones and a digital frequency analysis system. American Society for Testing and Materials ASTM E1050-10.

14. Anonymous. Standard test method for flexible cellular materials - slab, bonded, and molded urethane foams. American Society for Testing and Materials ASTM D3574.

15. Mariez E, Sahraoui S, Allard JF, Elastic constants of polyurethane foam's skeleton for Biot model, in: Proceedings of Internoise 1996: 951-954. 
16. Langlois C, Panneton R, and Atalla N. Polynomial relations for quasi-static mechanical characterization of isotropic poroelastic materials. J. Acoust. Soc. Am. 2001; 110: 3032.

17. Pritz T. Dynamic Young's modulus and loss factor of plastic foams for impact sound isolation. J. Sound. Vib. 1994; 178(3): 315-322.

18. D.L. Johnson, J. Koplik, and R. Dashen, "Theory of dynamic permeability and tortuosity in fluid-saturated porous media,” J. Fluid Mech. 1987; 176: 379.

19. Y. Champoux and J.-F. Allard, "Dynamic tortuosity and bulk modulus in air-saturated porous media,” J. Appl. Phys. 1991; 70(4).

20. Allard JF, Atalla N. Propagation of sound in porous media: Modeling sound absorbing materials, second ed. Willey; 2009.

21. Anonymous. Acoustics - Materials for acoustical applications - Determination of the airflow resistance”, International standard ISO 9053:1991(E).

22. Fellah ZEA, Berger S, Lauriks W, Depollier C, Aristégui C, Chapelon JY. Measuring the porosity and the tortuosity of porous materials via reflected waves at oblique incidence. J. Acoust. Soc. Am. 2003; 113(5): 2424.

23. U. Ingard, Sound absorption technology, Noise Control Foundation, New York; 1994.

24. Castagnède B, Aknine A, Brouard B, Tarnow V. Effects of compression on the sound absorption of fibrous materials. Appl. Acoust. 2000; 61(2): 173-82.

25. Campolina B, Dauchez N, Atalla N, Doutres O. Effect of porous material compression on the sound transmission of a covered single leaf panel. Appl. Acoust. 2012; 73, 791797. 
26. Anonymous. Standard test method for sound absorption and sound absorption coefficient by the reverberation room method. American Society for Testing and Materials ASTM C423-02. 


\section{Figure Captions}

Figure 1: Scheme of the shock tube and pictures of the experimental setup and material microstructure.

Figure 2: Photo-micrographs of the foams N1 and N2 and Scanning electron-micrograph of the foam N4; first column, before reticulation (blank); second column, after thermal reticulation; third column, after shock reticulation.

Figure 3: Scanning electron-micrographs of the polyimide foam N7; (a)-(b) before reticulation; (c)-(d) after shock reticulation. The shock treatment is carried out with the Mach number $\mathrm{M}_{\mathrm{s}}=1.62$.

Figure 4: Influence of the shock treatment strength on the polyurethane material N5: (a) sample thickness, (b) foam density, (c) airflow resistivity.

Figure 5: Influence of the shock treatment strength on the polyimide material N7: (a) sample thickness, (b) foam density, (c) airflow resistivity, (d) tortuosity, (e) Young's modulus.

Figure 6: Influence of the shock treatment strength on the sound absorption coefficient: (a) polyurethane material N5, (b) polyimide material N7. The base materials are 1 in. thick. The thickness of the reticulated samples can be smaller due to a plastic deformation. 
Table 1. Sound absorption average SAA [26] of polyurethane foam N5 and polyimide foam N7 for various shock treatments with increasing shock strength (the thickness of the samples varies according to Figs. 4(a) and 5(a)).

\begin{tabular}{|c|c|c|c|}
\hline \multicolumn{2}{|c|}{ polyurethane foam N5 } & \multicolumn{2}{|c|}{ polyimide foam N7 } \\
\hline$M_{s}$ & SAA & $M_{s}$ & SAA \\
\hline 1 & 0.49 & 1 & 0.24 \\
\hline 1.35 & 0.52 & 1.46 & 0.39 \\
\hline 1.50 & 0.50 & 1.58 & 0.32 \\
\hline 1.69 & 0.44 & 1.68 & 0.26 \\
\hline 1.85 & 0.40 & 1.81 & 0.20 \\
\hline
\end{tabular}


Table 2. Airflow resistivity $\sigma$ (in N.s.m ${ }^{-4}$ ) of 6 polyurethane foams N1-N6 and 1 polyimide foam N7 before and after being reticulated. The shock treatment is performed on both sides of the samples with a Mach number of 1.8 .

\begin{tabular}{|c|c|c|c|}
\hline Material & No treatment & Thermal reticulation & Shock reticulation \\
\hline N1 & 30000 & 270 & 530 \\
\hline N2 & 25000 & 170 & 380 \\
\hline N3 & 13000 & 2840 & 5370 \\
\hline N4 & 144500 & 6820 & 14200 \\
\hline N5 & 94370 & - & 15980 \\
\hline N6 & 96290 & - & 23620 \\
\hline N7 & 845300 & - & 22400 \\
\hline
\end{tabular}

